UVX 2010 (2011) 69-75

DOI: $10.1051 / \mathrm{uvx} / 2011010$

(C) Owned by the authors, published by EDP Sciences, 2011

\title{
Approche couplée pour le développement de matériaux optiques résistants aux radiations
}

\author{
S. Girard ${ }^{1}$, Y. Ouerdane ${ }^{2}$, N. Richard ${ }^{1}$, A. Boukenter ${ }^{2}$, M. Cannas $^{3}$ \\ et R. Boscaino ${ }^{3}$ \\ ${ }^{1}$ CEA, DAM, DIF, 91297 Arpajon, France \\ 2 Université de Saint-Étienne, Laboratoire Hubert Curien, 42000 Saint-Étienne, France \\ ${ }^{3}$ Università di Palermo, Laboratory of Amorphous Materials, Palerme, Italy
}

\begin{abstract}
Résumé. Nous présentons l'approche couplant simulations et expériences que nous avons mise en place entre le CEA et les Universités de Saint-Etienne et Palerme afin de prédire le comportement de matériaux optiques sous irradiation. La démarche suivie est illustrée en prenant pour exemples les résultats que nous avons obtenus autour de la thématique de la vulnérabilité des fibres optiques en environnement radiatif. Cette approche est notamment basée sur la définition d'échantillons canoniques: jeux de préformes, de fibres optiques et de cellules de simulation de grande taille conçus pour permettre la validation la plus directe possible des codes de calcul par l'expérience. Les principales avancées de notre étude sont présentées.
\end{abstract}

\section{INTRODUCTION}

L'intégration de technologies optiques est de plus en plus envisagée dans les différents domaines du nucléaire civil, spatial ou militaire. Certaines des applications visées doivent rester opérationnelles au sein des environnements extrêmes qui leur sont associés, notamment à de fortes contraintes radiatives (neutrons, photons gamma ou X, ions lourds,...). Parmi les technologies optiques, l'intégration de fibres optiques est à l'étude dans de nombreux projets tels que le projet Laser Mégajoule, ITER, des missions spatiales pour des applications de communications haut-débits, diagnostics plasma ou laser, capteurs à fibres optiques ou applications de contrôle commande [1-3]. Si les fibres optiques possèdent de nombreux avantages par rapport aux autres technologies (par ex: leur immunité électromagnétique, leurs faibles poids et volume, la possibilité de multiplexage, ... ), elles restent affectées par les radiations. $\mathrm{Au}$ niveau macroscopique, trois phénomènes principaux altèrent les propriétés des fibres optiques: 1) l'atténuation induite par irradiation qui entraîne un affaiblissement des capacités de transmission du guide d'onde, 2) l'émission induite sous irradiation qui, en se surimposant au signal utile, dégrade le rapport signal sur bruit et peut dans certains cas éblouir le détecteur 3) la compaction, généralement observée pour de fortes doses d'irradiation qui, en altérant les propriétés de la silice constituant la fibre optique, modifie ses propriétés de guidage. Ces effets s'expliquent par les propriétés structurelles, énergétiques et optiques de défauts ponctuels à l'échelle nanométrique ou sub-nanométrique dans la silice vitreuse qui sont induits par irradiation par des processus d'ionisation ou de déplacements atomiques.

Compte tenu de la multiplicité des paramètres influençant la génération de ces défauts et par extension la réponse radiative des fibres optiques (nature des dopants en coeur, en gaine, procédés de fabrication, profil d'emploi de la fibre, ... ) [1-6] et de la connaissance limitée de la définition des fibres optiques et verres commerciaux, il n'existe actuellement pas de modèle prédictif permettant, à

This is an Open Access article distributed under the terms of the Creative Commons Attribution-Noncommercial License 3.0, which permits unrestricted use, distribution, and reproduction in any noncommercial medium, provided the original work is properly cited. 
partir d'une structure de fibre optique donnée, d'estimer sa vulnérabilité pour une application donnée; ou à l'inverse, pour un environnement donné de déterminer la structure de fibre optique la plus adaptée pour l'application visée. Les modèles existants permettent uniquement, à partir de la caractérisation radiative d'une fibre optique, à l'aide de tests accélérés, d'estimer sa sensibilité à l'environnement réel de l'application [7-10].

Il est clairement établi que l'ensemble des structures et mécanismes de génération des défauts ponctuels dans les fibres optiques et verres de silice ne peuvent pas être appréhendés à partir d'études expérimentales seules. En effet, plusieurs phénomènes s'opposent à une telle identification systématique :

- La méconnaissance des échantillons expérimentaux - Tant la composition de leurs fibres optiques que leur procédé de fabrication sont considérés comme confidentiels par leurs fabricants ce qui interdit l'usage de fibres optiques commerciales pour notre étude. De plus, il convient de tenir compte de l'influence du procédé de fabrication qui ne peut être pris en compte qu'en étudiant simultanément les fibres optiques et préformes correspondantes.

- La complexité des phénomènes mis en jeu dans les fibres optiques commerciales - Les analyses chimiques révèlent la présence de codopants additionnels à la présence du germanium $(\mathrm{Ge})$ en cœur tels que le Fluor (F), le Phosphore (P) ou d'impuretés variant avec le procédé de fabrication (Chlore). L'étude doit donc être menée sur des échantillons simplifiés ou canoniques. Ceux-ci permettront d'identifier dans un premier temps l'influence propre à chacun des dopants et de valider les codes de calcul sur ces structures modèles et dans un second temps de traiter les verres complexes (multidopants) et les phénomènes d'interaction entre les dopants.

- Les limitations intrinsèques aux différentes techniques de mesure. L'association entre une structure du défaut et ses propriétés optiques (absorption, luminescence) ne peut être obtenue qu'en combinant différentes techniques possédant chacune des limites intrinsèques. Par exemple, la résonance paramagnétique électronique (RPE) ne permet d'investiguer que les défauts paramagnétiques, .... Une autre limitation forte associée aux techniques de mesures expérimentales reste, pour certaines telles que la RPE, l'impossibilité de faire des mesures en ligne lors de l'irradiation.

Ceci explique que, malgré des décennies de recherche expérimentale, la nature et les propriétés de tous les défauts n'aient pas pu encore être clairement identifiées. L'outil de simulation quant à lui permettra à terme d'étudier chaque mécanisme de génération et structure de défaut de manière isolée et devrait permettre de franchir un pas significatif vers une compréhension plus complète des effets des radiations dans les verres. Cependant, à l'heure actuelle, les outils théoriques nécessaires doivent encore être améliorés ou même dans certains cas créés. Leur validation ne pourra se faire que par la confrontation aux résultats expérimentaux. Conjointement, l'augmentation constante des capacités de calcul des supercalculateurs permet aujourd'hui d'envisager l'étude par des techniques ab initio des cellules de simulation de taille suffisante pour être représentatives des verres réels.

La présentation de notre approche et des premiers résultats obtenus a été publiée en 2008 sous la forme de deux articles complémentaires [11,12]. L'obtention d'un tel outil de prédiction nécessite en effet de travailler à de nombreux niveaux (cf Fig. 1a) depuis l'échelle atomique des défauts ponctuels jusqu'à la réponse macroscopique du verre pour un profil d'emploi et un environnement radiatif donnés.

La simulation doit tout d'abord être appliquée à l'échelle atomique et inter-atomique (échelle 1) afin de modéliser les mécanismes de génération des défauts ponctuels dans les verres. Il convient d'être capable de calculer correctement les propriétés énergétiques, structurales et optiques de ces défauts ainsi que leur distribution liée à la nature amorphe du verre. En nous appuyant sur la puissance de calcul du supercalculateur du CEA DAM, nous avons montré que la théorie choisie, la DFT (Density Functional Theory) [13], permettait de simuler efficacement le caractère amorphe de verre de silice pur ou dopé ainsi que ses propriétés structurales sur des cellules de 108 atomes. En revanche, d'autres théories plus sophistiquées, au-delà de la théorie de la fonctionnelle de la densité, sont nécessaires pour calculer les bonnes propriétés électroniques et optiques des défauts telles que l'approximation dite GW et l'équation de Bethe Salpeter [14]. 

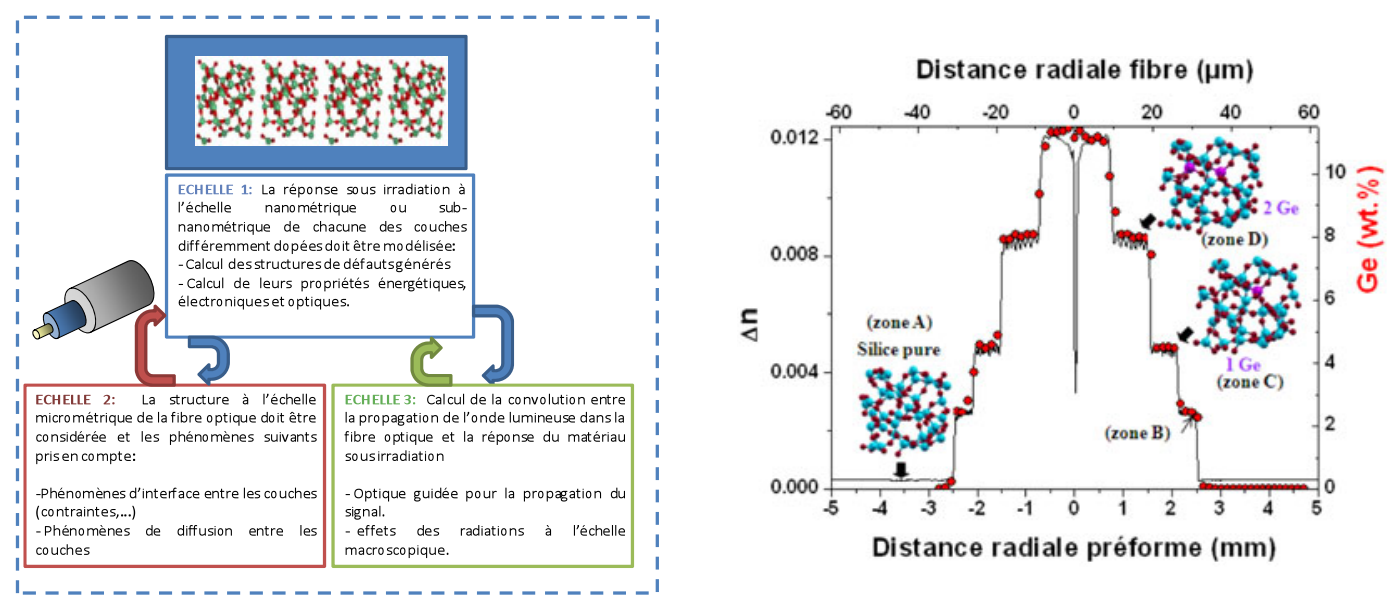

Figure 1. (a) Représentation schématique de l'outil théorique de prédiction du comportement des fibres optiques sous irradiation (b) Distribution radiale de germanium (mesurée par microsonde électronique) dans la fibre canonique Ge, son profil d'indice mesuré à $633 \mathrm{~nm}$. Correspondance entre la composition des zones $\mathrm{A}, \mathrm{C}$ et $\mathrm{D}$ et les cellules de simulation de 108 atomes contenant respectivement 0,1 et 2 atomes de Ge utilisées pour les calculs ab initio.

Le niveau suivant (échelle 2) consistera à prendre en compte les possibles interactions entre les différentes couches de verre constituant le cœur et la gaine de la fibre optique et leurs conséquences sur la réponse du matériau. Cette étape requiert la capacité de modéliser les mécanismes de diffusion de charges, de migration d'éléments chimiques ainsi que la reproduction de l'historique du verre (états d'interface entre les couches, répartition des contraintes dans la structure, ...). Enfin, il convient ensuite de simuler les effets macroscopiques des radiations sur la fibre optique ou le verre considérés (échelle 3). Pour cela, il faudra pouvoir convoluer la réponse des différents matériaux constituant la fibre optique à la propagation de l'onde lumineuse représentant le signal utile.

\section{PRÉSENTATION DES ÉCHANTILLONS CANONIQUES}

Notre approche ne pouvant pas être menée sur des échantillons commerciaux de verre et fibres optiques, nous avons choisi de développer nos propres échantillons, dénommés ci-après échantillons canoniques. Ils sont constitués, d'une part par des échantillons expérimentaux de fibres optiques et de préformes fabriquées par la société iXFiber via le procédé Modified Chemical Vapor Deposition (MCVD) [15], et d'autre part par des cellules de simulation de taille importante ( $>100$ atomes) utilisées pour les calculs $a b$ initio. Les contraintes liées à la partie théorique étant assez importantes (la taille maximale des cellules reste limitée par la puissance des supercalculateurs), nous avons choisi de faire développer des verres de silice pure et dopée possédant des caractéristiques proches en termes de composition des cellules de simulation les plus adaptées à nos calculs théoriques. Aujourd'hui, nous avons développé six types d'échantillons canoniques, décrits dans la table 1.

Dans un premier temps, une série de trois échantillons canoniques a été créée afin d'étudier qualitativement et quantitativement l'influence des trois principaux dopants: Ge, P, F. Il convient de noter que la réponse de la silice pure a pu être pré évaluée en parallèle en étudiant la gaine optique de ces échantillons. Les mesures expérimentales sur ces trois premiers échantillons sont ensuite comparées aux calculs menés à l'échelle 1. Ensuite, afin de tenir compte des nouveaux instruments de mesure accessibles à travers la collaboration et d'investiguer quantitativement l'influence du procédé d'étirage de la fibre optique sous irradiation, trois autres échantillons canoniques à base de silice dopée $\mathrm{F}, \mathrm{Ge}$ et silice pure ont été fabriqués en faisant varier les conditions d'étirage pour l'obtention des fibres 
Tableau 1. Description sommaire des échantillons canoniques développés pour l'approche couplée.

\begin{tabular}{|c|c|c|c|c|}
\hline $\begin{array}{l}\text { Ech } \\
\text { canonique }\end{array}$ & Composition cœur & Composition Gaine & Conditions d'étirage & $\begin{array}{l}\text { Publications } \\
\text { associées }\end{array}$ \\
\hline$\# 1 \_G e$ & Silice dopée Ge & Silice pure & standard & {$[11,12,16-18]$} \\
\hline$\# 2 \_F$ & Silice dopée F & Silice dopée F & standard & {$[11,19,20]$} \\
\hline \#3_P & Silice dopée P & Silice pure & standard & {$[21]$} \\
\hline \#4_F-D & Silice dopée F & Silice dopée F & Froid, standard, chaud & \\
\hline \#5_Ge-D & Silice dopée $\mathrm{Ge}$ & Silice pure & Froid, standard, chaud & \\
\hline \#6_SiO2 & Silice pure & Silice dopée F & Froid, standard, chaud & \\
\hline
\end{tabular}

optiques. L'objectif est ici de traiter du point de vue expérimental, les différents phénomènes qu'il faudra modéliser à l'échelle 2 pour développer l'outil de prédiction. Il est important de préciser que nous avons vérifié que ces échantillons sont représentatifs au regard des fibres optiques commerciales fabriquées par MCVD tant en terme de composition que de réponse sous irradiation.

Un autre point très important dictant la conception de ces guides d'onde est leur capacité à reproduire les cellules de simulation développées pour les calculs ab initio. Cet aspect est illustré sur la figure $1 \mathrm{~b}$ qui représente les distributions radiales du profil d'indice et de la concentration en Ge que nous avons choisies pour notre échantillon canonique. Le profil d'indice de ce guide d'onde a été choisi volontairement très différent de celui des fibres optiques commerciales à saut (SI) ou à gradient d'indice (GI). Nous avons choisi de créer ce verre avec différents paliers de dopage en Ge depuis une gaine optique (zone $\mathrm{A}$ en silice pure, $0 \%$ de $\mathrm{Ge}$ ) jusqu'à un palier central dopé avec $11,5 \%$ de Ge. Cette structure de fibre optique possède plusieurs avantages pour notre approche. Elle permet, lorsque l'on dispose d'outils résolus spatialement, de quantifier l'influence de la concentration en Ge sur la réponse de la silice sous irradiation mais aussi d'obtenir dans un seul échantillon des compositions comparables à des cellules de simulation à différents niveaux de dopage. Ainsi pour la fibre illustrée sur la figure $1 \mathrm{~b}$, l'étude de la réponse de la zone A permet de comparer les mesures expérimentales aux calculs théoriques dans la cellule de silice pure, la zone $\mathrm{C}$ les mesures et calculs de la cellule de 108 atomes contenant un atome de Ge et la zone D celle de la cellule avec 2 atomes de Ge.

Pour chacun des échantillons canoniques, nous avons pu mener les différents essais sous irradiations transitoires et continues ainsi que les études spectroscopiques post mortem, l'objectif final étant de comparer les résultats théoriques et expérimentaux et d'identifier les mécanismes à l'origine de la génération des défauts dans les verres. Les publications issues de la caractérisation de ces échantillons sont listées dans le tableau 1.

\section{MÉTHODOLOGIE DE SIMULATION}

La méthodologie de simulation, schématiquement représentée dans la figure 2 est brièvement explicitée dans ce paragraphe. Plus de détails concernant les calculs théoriques peuvent être trouvés dans les publications issues de nos travaux et dans les références citées dans ces articles [12,22,23]. L'objectif de ces calculs est dans un premier temps de montrer la faisabilité d'obtention des propriétés structurales, énergétiques et optiques des défauts ponctuels dans des verres de silice amorphe pure ou dopée, dans un second temps d'étudier l'influence de l'environnement local du défaut sur ses mécanismes de génération et enfin de valider notre méthodologie via la confrontation de nos calculs aux expérimentations menées sur les échantillons canoniques de fibre optique et de préforme.

\subsection{Génération des cellules de simulation}

La première étape consiste à générer des cellules de simulation de grande taille (108 atomes, $36 \mathrm{Si}$ et $72 \mathrm{O}$ ) représentatives des verres de silice pure par dynamique moléculaire classique (DM). La 
cellule est ensuite relaxée en ab initio dans le cadre de la théorie de la DFT avec le code PWSCF (Plane Wave Self Consistent Field) [12]. A l'heure actuelle, seule cette cellule a été étudiée par la simulation. L'incorporation des dopants et impuretés dans la silice, que ce soit le Germanium ou des éléments non tétravalents tels que le Fluor et le Phosphore, se fera dans un second temps, leur processus d'incorporation dans le verre devant être représentatif de celui utilisé pour doper les différentes couches de préforme par la technique MCVD. La représentativité de cette cellule par rapport à la silice amorphe a été validée par la comparaison de ses propriétés structurales et énergétiques avec les verres réels [12]. Les cellules dopées sont obtenues par remplacement d'un (ou de deux) atome(s) de silicium par du germanium dans la cellule. Les cellules sont alors relaxées afin d'obtenir la structure à l'équilibre. La génération des cellules contenant des défauts tels que des lacunes en oxygène, défauts Si-ODC(I) ou GeODC(I) (Si-X, X = Ge ou Si), est obtenue en enlevant un atome d'oxygène de la cellule et en laissant relaxer celle-ci. Afin d'évaluer l'influence de l'environnement local sur les propriétés de ce type de défaut, nous avons généré l'ensemble des Si-ODC et Ge-ODC possibles dans nos matrices en déplaçant le site contenant la lacune d'oxygène. Par cette technique, nous avons donc obtenu 72 cellules différentes contenant un Si-ODC et 144 cellules contenant un Ge-ODC. L'ionisation des cellules contenant des vacances d'oxygène est utilisée, selon le processus décrit dans [12], afin de générer des défauts chargés de type SiE' ou GeE' [1-3].

\subsection{Détermination des propriétés structurales et énergétiques}

La DFT permet d'accéder à un ensemble important de données concernant les propriétés structurales et énergétiques des ODC dans la silice amorphe. Ainsi, il est possible de calculer les énergies de formation

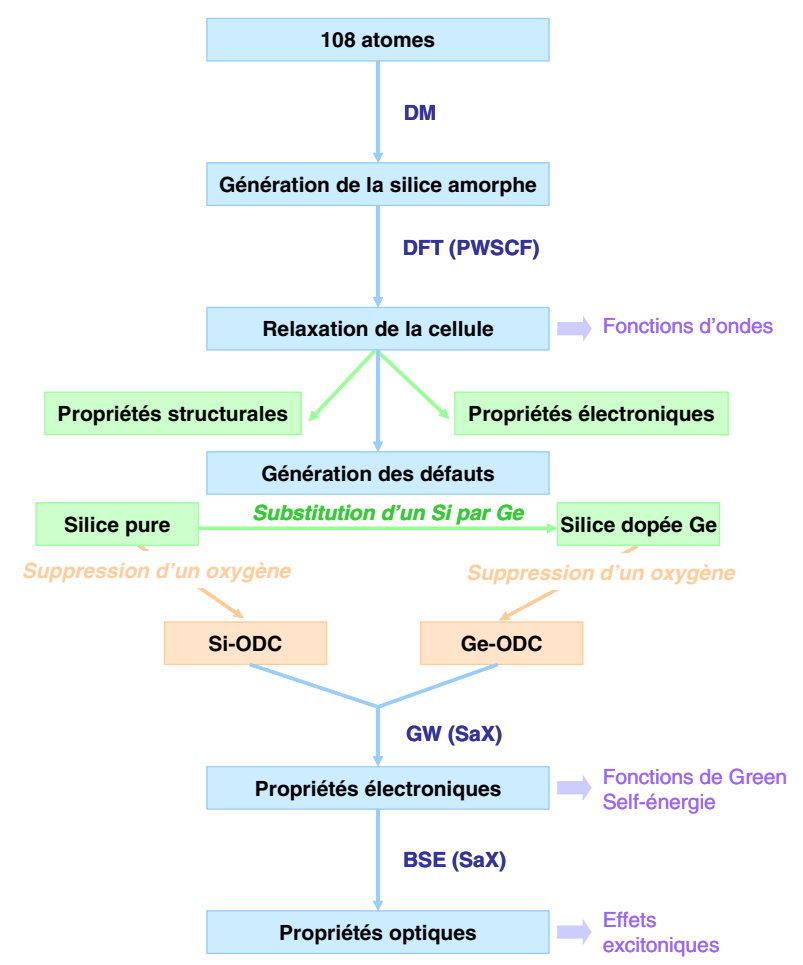

Figure 2. Représentation schématique de la démarche suivie pour le calcul des propriétés structurales, énergétiques et optiques dans les verres de silice pure et dopée Ge: exemple des défauts ODC (déficients en oxygène). 
d'un défaut pour les différents sites possibles dans la cellule afin d'estimer l'efficacité de sa génération en fonction de l'environnement local. Ses limitations intrinsèques ne permettent pas en revanche de l'utiliser pour obtenir un calcul correct des propriétés électroniques et optiques de ces défauts. Cette limitation est due au fait que la DFT est intrinsèquement une théorie d'état fondamental et ne prend donc pas en compte les états excités, ce qui d'un point de vue pratique entraîne une sous-estimation systématique de la largeur de la bande interdite.

\subsection{Détermination des propriétés électroniques et optiques}

Le calcul des propriétés électroniques et optiques implique d'utiliser une théorie allant au-delà de la DFT prenant en compte les excitations électroniques. Ceci est réalisé en utilisant des méthodes qui introduisent une meilleure prise en compte du problème à plusieurs corps. Elles sont introduites comme des perturbations à la DFT au sens de la mécanique quantique. Nous nous basons sur les résultats DFT pour relancer ces calculs. Pour ceux-ci, nous utilisons le code Sax [24]. Ce code s'interface directement avec le code PWSCF. Un premier niveau est l'approximation GW que nous utilisons pour obtenir les propriétés électroniques de nos défauts ponctuels. L'obtention des propriétés optiques, dont les propriétés d'absorption par exemple, nécessite une étape supplémentaire dans la théorie, qui passe par la résolution de l'Equation de Bethe Salpeter traitant les états excitoniques. Ces outils ont été validés par le bon accord entre le calcul de la bande d'absorption associée aux SiODC dans la cellule de simulation et les données expérimentales de la littérature.

\section{CONCLUSIONS}

Nous avons démontré à ce jour la pertinence de l'approche couplant simulation ab initio et expériences que nous avons mise en place entre le CEA et les Universités de Saint-Etienne et Palerme. Les perspectives concernent l'obtention d'une base de données établie par le calcul contenant les propriétés optiques des différents défauts ponctuels liés aux principaux dopants tels que le $\mathrm{Ge}, \mathrm{P}$ ou F. A plus long terme, les interactions entre ces dopants et leurs interactions avec des impuretés tels que le chlore seront également traitées. Enfin, le développement d'outils de simulation multi-échelle sera nécessaire afin de remonter à l'influence de ces défauts ponctuels à l'échelle macroscopique.

\section{Références}

[1] S. Girard, "Analyse de la réponse des fibres optiques soumises à divers environnements radiatifs", Thèse de doctorat, Université de Saint-Etienne, 2003.

[2] M. Van Uffelen, "Modélisation de systèmes d'acquisition et de transmission à fibres optiques destinés à fonctionner en environnement nucléaire", Thèse de doctorat, Université Paris XI, 2001.

[3] B. Brichard, "Systèmes à fibres optiques pour infrastructures nucléaires: du durcissement aux radiations à l'application" Thèse de doctorat, IES - Institut d'Electronique du Sud, Montpellier, 2008.

[4] S. Girard, J. Keurinck, A. Boukenter, J.-P. Meunier, Y. Ouerdane, B. Azaïs, P. Charre, and M. Vié Nuc. Instr. and Meth. B 215 (2004) 187-195.

[5] S. Girard, J. Keurinck, Y. Ouerdane, J.-P. Meunier, and A. Boukenter, IEEE/OSA J. Light. Techn. 22 (2004) 1915-1922.

[6] S. Girard, Y. Ouerdane, A. Boukenter, and J.-P. Meunier, J. of Appl. Phys.. 99 (2006) 023104.

[7] D.L. Griscom, M.E. Gingerich, E.J. Friebele, Phys. Rev. Letters, 71 (1993) 1019-1022.

[8] D. L. Griscom, J. Non-Cryst. Solids 352 (2006) 2601-2617.

[9] M. Caussanel, "Tenue aux rayonnements de composants optoélectroniques destinés à l'environnement spatial - L'amplificateur optique à fibre dopée Erbium", Thèse de doctorat, IES - Institut d'Electronique du Sud, Montpellier, 2004. 
[10] P. Borgermans, "Spectral and Kinetic Analysis of Radiation Induced Optical Attenuation in Silica: Towards Intrinsic Fiber Optic Dosimetry?”, Thèse de doctorat, Brussel, Vrije Universiteit, 2001.

[11] S. Girard, Y. Ouerdane, G. Origlio, C. Marcandella, A. Boukenter, N. Richard, J. Baggio, P. Paillet, M. Cannas, J. Bisutti, J-P. Meunier and R. Boscaino, IEEE Trans. Nucl. Sci. 55 (2008) 3743-3482.

[12] S. Girard, N. Richard, Y. Ouerdane, G. Origlio, A. Boukenter, L. Martin-Samos, P. Paillet, J-P. Meunier, J. Baggio, M. Cannas, R. Boscaino IEEE Trans. Nucl. Sci. 55 (2008) 3508-3514.

[13] P. Hohenberg et W. Kohn, Phys. Rev. B, 136 (1964) 864; W. Kohn et L.J. Sham, Phys. Rev. A, 140 (1965) 1133.

[14] voir revue dans Giovanni Onida, Lucia Reining et Angel Rubio, Rev. Mod. Phys. 74 (2002) 601

[15] iXfiber website: www.ixfiber.com/

[16] G. Origlio, S. Girard, M. Cannas, Y. Ouerdane, R. Boscaino, and A. Boukenter, J. Non-Cryst. Solids 355 (2009) 1054-1056.

[17] G. Origlio, M. Cannas, S. Girard, R. Boscaino, A. Boukenter, Y. Ouerdane, Optics Letters 34 (2009) 2282-2284.

[18] G. Origlio, S. Girard, R. Boscaino, A. Boukenter, M. Cannas, and Y. Ouerdane, EDP Sciences, (2009) 127-132.

[19] S. Girard, C. Marcandella, G. Origlio, Y. Ouerdane, A. Boukenter, J.-P. Meunier, J. Non-Cryst. Solids 355 (2009) 1089-1091.

[20] G. Origlio, A. Boukenter, S. Girard, M. Cannas, R. Boscaino, Y. Ouerdane, Nuc. Instr. and Meth. B 266 (2008) 2918-2922.

[21] G. Origlio, F. Messina, M. Cannas, R. Boscaino, S. Girard, A. Boukenter, Y. Ouerdane, Physical Review B 80 (2009) 205208-1.

[22] N. Richard, S. Girard, L. Martin-Samos, A. Boukenter, Y. Ouerdane and J-P. Meunier, Nuc. Instr. and Meth. B 266 (2008) 2719-2722.

[23] Y. Laudernet, N. Richard, S. Girard, L. Martin-Samos, A. Boukenter, Y. Ouerdane, and J.-P. Meunier, J. Non-Cryst. Solids 352, (2006) 2596-2600.

[24] Sax website: http://www.s3.infm.it/sax/ 